\title{
Evidence and Considerations on Treatment of Small Size Merkel Cell Head and Neck Carcinoma
}

\author{
Elena Festa Kotelnikova1( ${ }^{10}$ Melissa Laus ${ }^{2(1)}$ Adelchi Croce $^{1(0)}$ \\ 1 Department of ENT, University “G. d'Annunzio" of Chieti-Pescara, \\ Hospital "SS Annunziata," Chieti, Italy \\ 2 Department of Otolaryngology, General Hospital "S. Giovanni \\ Calibita - Fatebenefratelli”, the Tiber Island, Rome, Italy \\ Address for correspondence Laus Melissa, MD, Department of \\ Otolaryngology, General Hospital "S. Giovanni Calibita - \\ Fatebenefratelli," the Tiber Island, Rome, Italy \\ (e-mail: melissa.laus@hotmail.it).
}

Int Arch Otorhinolaryngol 2020;24(4):e487-e491.

\begin{abstract}
Introduction Merkel cell carcinoma (MCC) is a rare and aggressive neuroendocrine malignant cancer. It is an epidermal cancer common in the head and neck.

Objectives Though there is limited number of cases described in the literature for the treatment difficult to obtain. Our purpose was to present the clinical course and treatment of four patients with MCC.

Methods We conducted a retrospective analysis and obtained detailed clinical information for all 4 patients treated for MCC at the ENT Department of the SS Annunziata Hospital in Chieti, Italy, from 2013 through 2015.

Results In our study, two patients presented with the tumor in a rare site (lower eyelid). All of the patients underwent surgical treatment: three patients had free excision margins and negative sentinel lymph nodes (SLNs) while 1 patient had free

Keywords

- merkel cell carcinoma

- merkel cell carcinoma treatment

- neuroendocrine tumor

- rare tumor

- head and neck cancer excision margins and positive SLNs. The latter patient underwent ipsilateral neck dissection. In another patient, the fluorodeoxyglucose positron emission topography (FDG PET)/computed tomography (CT) performed 6 months after the surgery has shown high metabolic activity in the left parotid gland, and the patient underwent total parotidectomy and a neck dissection.

Conclusion Sentinel lymph node biopsy is a useful technique in small size MCCs of the head and neck. However, the parotid gland should be strictly controlled in patients with lower eyelid tumors.
\end{abstract}

\section{Introduction}

Merkel cell carcinoma (MCC) is a rare and highly aggressive cutaneous-neuroendocrine tumor that presents as a rapidly growing, solitary, cutaneous or subcutaneous, pink-to-violet nodule located mostly on sun-exposed areas.

The MCC prognosis is considered as bad as that of melanoma. The incidence rate ranges from 0.13 per 100,000 people in Europe (between 1995 and 2002) to 1.6 per 100,000 people in Australia (between 1993 and 2010). ${ }^{1-4}$ The average age at the time of diagnosis is 76 years old. At the primary diagnosis, loco-regional metastases are

received

December 16, 2018

accepted

January 26, 2020
DOI https://doi.org/

10.1055/s-0040-1709114. ISSN $1809-9777$. already present in $\sim 30 \%$ of the patients, and the rate of local recurrence after treatment is of between 40 and $77 \%$. Metastases are localized in the skin (28\%), lymph nodes (27\%), the liver (13\%), the lungs (10\%), bones $(10 \%)$ and the brain $(6 \%)$, and the estimated mortality rate is between 33 and $46 \%$. $1,3,6,7$

Merkel cell carcinoma takes its name from the small grains of the tumor cells similar to the grains of the Merkel cells. The markers are neuron specific enolase, chromogranin, synaptophysin and CD56, the same of pulmonary microcytoma, and cytokeratin 20.
Copyright @ 2020 by Thieme Revinter Publicações Ltda, Rio de Janeiro, Brazil
License terms

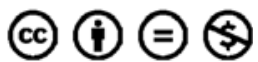


Risk factors for MCC are ultraviolet (UV) radiation exposure, fair skin, advanced age and immune suppression (i.e., patients with organ transplantation, lymphoproliferative diseases, HIV, immunosuppressive therapy for autoimmune diseases).

The main hypotheses about the etiology of MCC are Merkel cell polyomavirus (MCPyV) integration in MCC cells (detected in 43 to100\% of patient samples) and accumulation of UV-induced mutations in the MCPyV-negative MCCs. ${ }^{1,8,9}$

The rarity of MCC and lack of comprehensive randomized control studies hampers providing answers to all of the questions of the clinicians involved in the treatment of this tumor.

We examined 4 cases of small size MCC localized in the face with the aim of determine some features that may help in understanding the influence of MCC localization, MCC size and patients comorbidities on the treatment and follow-up choices.

\section{Method}

We examined 4 patients who arrived at the ENT Department of our Hospital with a diagnosis of cutaneous MCC between August 2013 and June 2015. The patients age ranged from 68 to 88 years old (mean age of 75.75 years old).

The MCCs were localized in the upper part of the face (lower eyelid in 2 patients, eyebrow in 1 patient, auricle in 1 patient). The diagnosis was made via an excisional biopsy with close margins performed in other hospitals a few weeks before.

The MCCs were initially staged according to the American Joint Committee on Cancer (AJCC) Tumor, lymph nodes and metastasis (TNM) Staging Classification for Merkel Cell Carcinoma $\left(7^{\text {th }}\right.$ ed., 2010). For the present study we retrospectively applied the most recently published AJCC TNM Staging Classification for Merkel Cell Carcinoma $\left(8^{\text {th }}\right.$ ed., 2016) staging criteria. ${ }^{10}$ All of the patients were $\mathrm{PT} 1 \mathrm{cNOMO}$ at presentation. In agreement with the National Comprehensive Cancer Network (NCCN) guidelines and with the clinical practice guidelines of our institute, we performed a wide local excision (WLE) of the lesion and a sentinel lymph node biopsy (SLNB).

All of the patients had follow-up visits (with clinical examination, neck ultrasound and whole body fluorodeoxyglucose positron emission topography (FDG PET)/computed tomography (CT) at least once a year, and magnetic resonance imaging (MRI) with contrast or CT with contrast in some clinical circumstances) scheduled every 3 to 6 months. All of the patients were free of disease at the latest follow-up visit.

Because of the small number of patients, statistical analysis was not performed for prognostic indications.

- Table 1 lists the patients (numbered 1-4) and the details of the treatments performed.

\section{Ethical Considerations}

All of the patients signed an informed consent form for the processing of personal data.

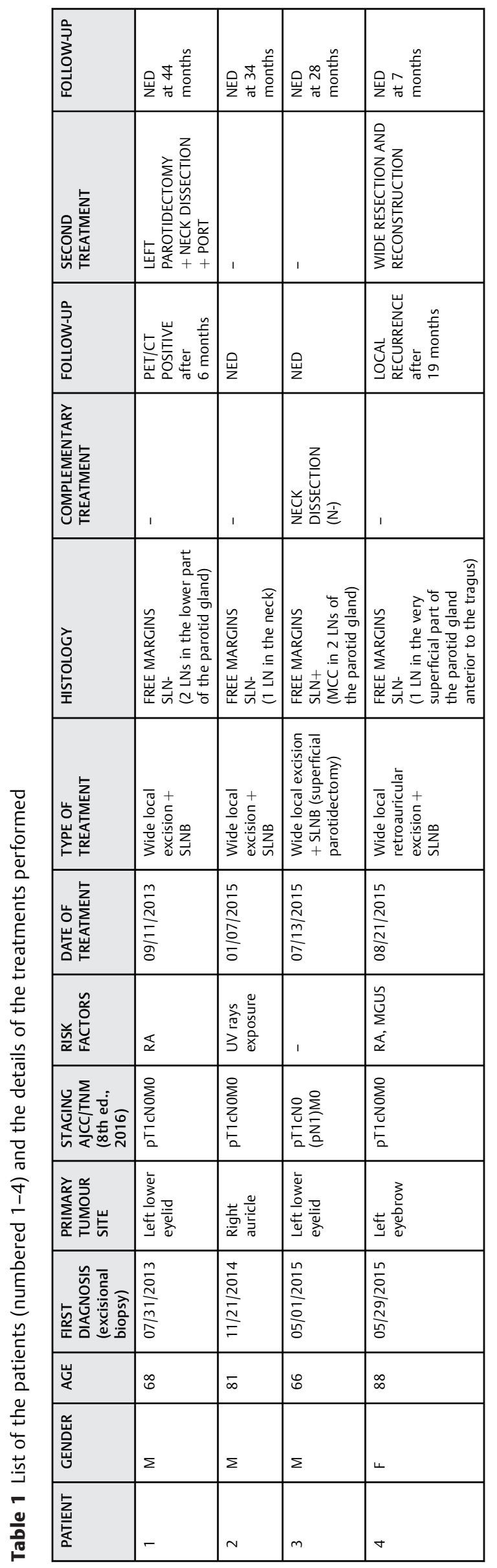




\section{Results}

\section{Case Presentation}

Case 1

A 68-year-old Caucasian man had an excisional biopsy of the inferior left eyelid resulting in MCC 42 days before. He had a history of tobacco use (> 20 cigarettes/day until 2003), stroke (10 years before), carotid artery stenosis and autoimmune disease (rheumatoid arthritis [RA]).

At the WLE and SLNB (2 lymph nodes [LNs] in the lower part of the parotid gland), he had free excision margins and negative sentinel lymph nodes (SLNs). He did not receive postoperative radiation therapy (PORT) because the SLNs were negative and the tumor was small, widely excised, and located near the eye.

In the follow-up, whole body FDG PET/CT performed 6 months after the surgery showed high metabolic activity in the left parotid gland. Then, a total left parotidectomy and an ipsilateral neck dissection were performed. The histological examination showed MCC localization in 1 parotid LN and in 1 (of 21 resected) neck LN. The patient underwent PORT as proposed by our multidisciplinary team. The patient is free of disease at 44 months of follow-up.

\section{Case 2}

An 81-year-old Caucasian man underwent an excisional biopsy of a right auricle lesion 47 days before. The immunohistochemistry confirmed the presence of an MCC with one positive margin without extension to the cartilage. He had a history of intensive UV exposure and aortic and mitralic valves insufficiency.

We performed a subtotal excision of the right auricle without reconstruction and the SLNB (the LN was located in level I). The patient did not receive PORT because the SLN was negative, the tumor was small and widely excised, and he had no other risk factors. The patient is free of disease at 34 months of follow-up.

\section{Case 3}

A 66-year-old Caucasian man had a biopsy of the left lower eyelid with a diagnosis of MCC 2 months before. The biopsy was performed 7 months after the onset of the macroscopic lesion.

The patient had a history of pulmonary embolism, myocardial infarction, type 2 diabetes.

We performed a wider excision of the lower left eyelid and a left superficial parotidectomy to remove the SLNs, located in the superficial lobe of the parotid gland. At the histological examination, the 2 SLNs had evidence of metastatic MCC, so we performed a neck dissection. Cervical lymph nodes resulted free of disease at the histological examination, and the patient refused PORT proposed by our multidisciplinary team. The patient is free of disease at 28 months of follow-up.

\section{Case 4}

An 88-year-old Caucasian woman underwent the excision of a left eyebrow lesion almost 3 months before with the unexpected diagnosis of MCC with positive deep margin of excision.
The patient had a history of autoimmune disorders (RA and monoclonal gammopathy of undetermined significance [MGUS]).

A wide excision of the eyebrow with forehead rotation flap reconstruction and the concomitant excision of the SLN, located in the very superficial part of the parotid gland, anterior to the tragus, were performed. Both specimens were negative for MCC. The patient did not receive PORT because the SLNs were negative, the tumor was small, widely excised, and located near the eye and, moreover, the patient was very old.

In the follow up, 19 months after the first surgery, we found 2 local recurrences beside the scar of the first surgery (-Fig. 1b, 1c). The neck and parotid ultrasound examination was negative. We performed a wide excision of the lesions and a local flap reconstruction with suspension of left supraorbital soft tissue to the underlying bone (-Fig. 1d). Considering the age of the patient and the location of the lesion, our multidisciplinary team decided on the observation of the primary tumor site without PORT or chemotherapy in spite of local recurrences. The patient is free of disease at 7 months of follow-up after the local recurrence, 26 months after the first surgery.

\section{Discussion}

From our case series, we can see that the patients affected by immune disorders had locoregional recurrences. Immune suppression is an accepted risk factor for MCC and our evidences underline the necessity for a very strict followup even for very small-sized MCCs in patients with immune system disorders.

Another observation is that the only patient with positive SLNs was the one with the longer interval between the onset of the lesion and the excision. This patient had no story of immune suppression and the primary lesion was a small size tumor. Indeed, another factor influencing the aggressivity of the tumor in patient 3 could be the patient age, since the positive SLNs were detected in the younger patient of our case series. Maybe further studies can suggest if the time from the onset of the lesion and the patient age can be relevant factors in the planning of the therapeutic strategy and follow-up.

Sentinel lymph node biopsy is recommended as the first step in clinically NO patients, ${ }^{10,11}$ since clinically occult micrometatases are present in $30 \%^{5}$ of them. Nevertheless, the reliability of SLNB in periocular region tumors is debated. ${ }^{12}$ Periocular MCC is very rare, representing from 5 to $20 \%$ of the head and neck MCCs. ${ }^{12-14}$ Much of what we assume about the behavior of the tumor and the role of SLNB at this site is extrapolated from MCCs at other anatomic locations and data from other eyelid and conjunctival tumors. These tumors are typically smaller, diagnosed earlier and localized in a unique site with a high degree of lymphatic drainage variability. $^{12}$

In our study, we considered 2 patients with this rare tumor site, located in both of them in the lower eyelid, both with a small tumor size $(<1 \mathrm{~cm})$. The SLNB was positive 

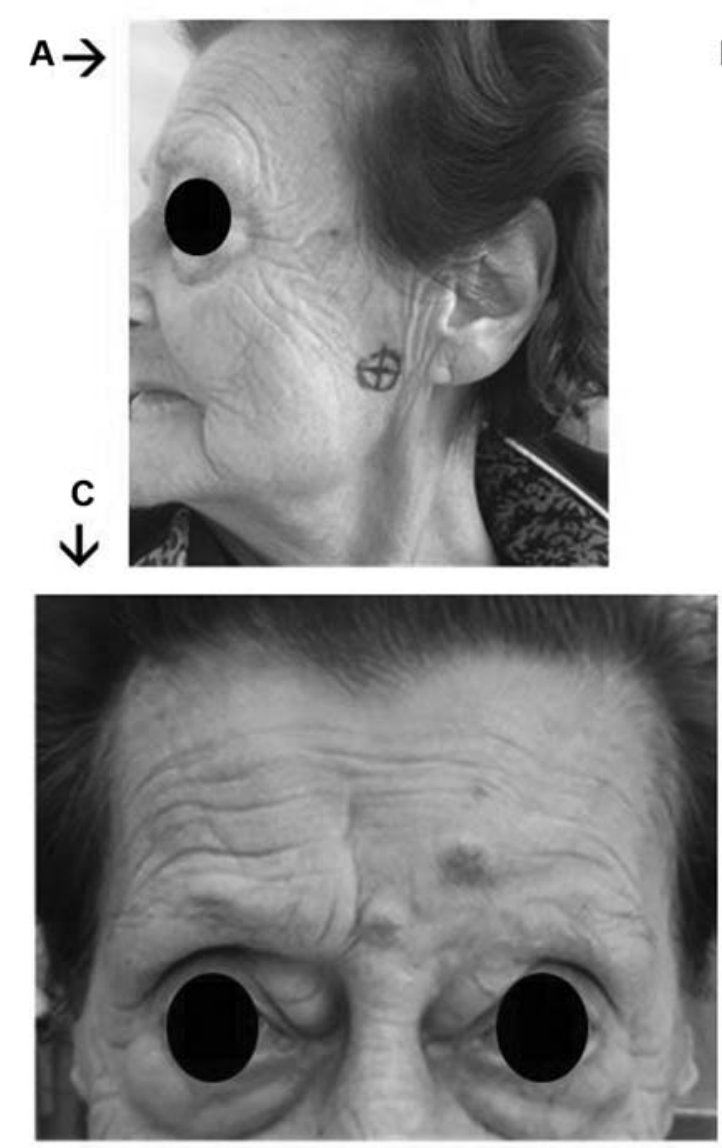
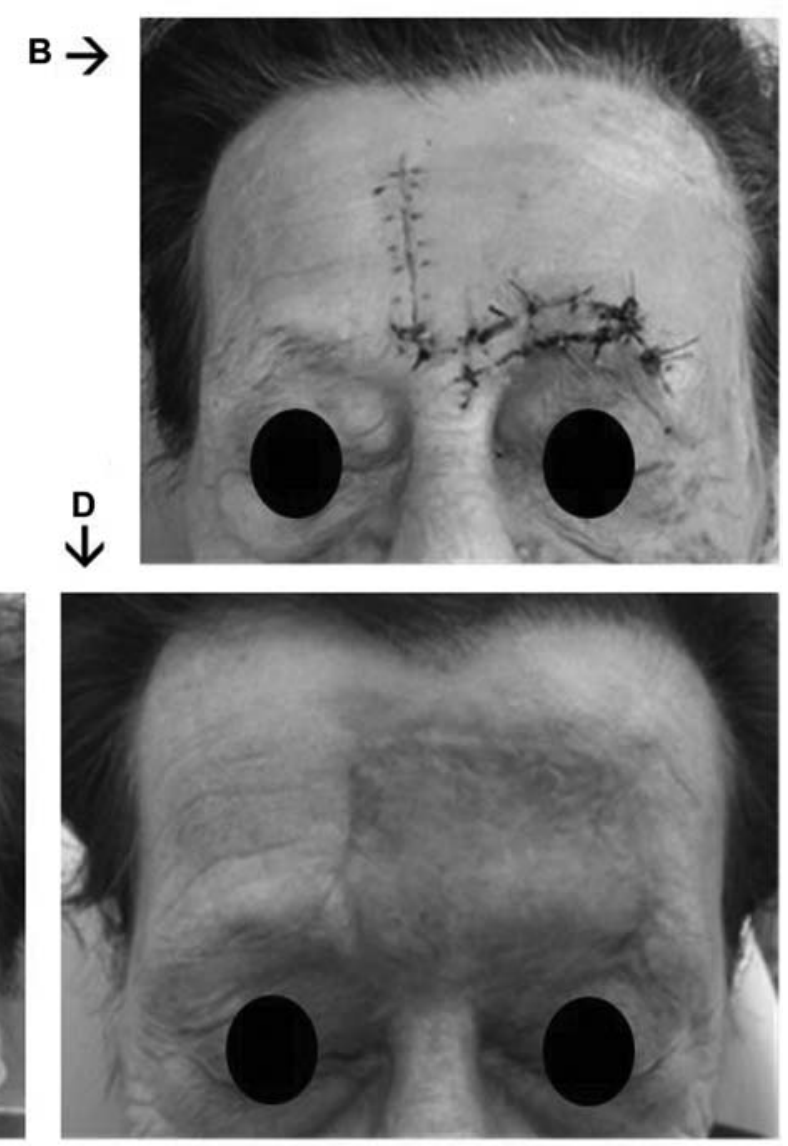

Fig. 1 Patient $4 \diamond$ (a) Sentinel lymph node location in patient 4; (b) Early postoperative aspect after removal of the lesion with wide margins and reconstruction with local flaps performed in the first surgery; (c) Local recurrence beside the scar of the first surgery; (d) Postoperative aspect after removal of the lesions with wide margins, flap and suspension.

in 1 of 2 patients (50\%), so despite the small number of patients, considering the rarity of the tumor, this positivity can be a valid datum in support of the usefulness of SLNB. Another observation is that both patients had a parotid gland localization after at least 7 months from the onset of the primary lesion (in patient 1 discovered 6 months after surgery, in patient 3 discovered with SLNB technique because of the 7-month delay of the primary treatment). Therefore, the parotid gland should be strictly monitored in patients with lower eyelid tumors and maybe, if supported by further studies, we could consider performing a superficial parotidectomy in these patients even if the SLN is located in the very peripheral part of the gland.

Many studies indicate that even smaller MCCs carry a significant risk of locoregional disease spread. Some authors demonstrated SLNB positivity rates of $23 \%$ in tumor sizes $\leq 1 \mathrm{~cm}$, others showed a similar $26 \%$ SLN positivity at $\leq 1 \mathrm{~cm}$ tumor size, and another demonstrated $14 \%$ nodal involvement for $0.5 \mathrm{~cm}$ tumors. ${ }^{12,15-17}$

All of the patients of our study had small size MCCs. Sentinel lymph node biopsy was a reliable technique in patients 1 and 3 for the reasons given above, and it was a reliable technique for the remaining patients (patients 2 and 4 ) because their negative SLNBs are confirmed by the clinically and radiologically negative neck for MCC localization, respectively, after 34 months and
26 months from the SLNB follow-up. Since between 80 and $90 \%$ of all MCC recurrences happen within 24 months (median 8 months), these data have an additional value. ${ }^{5,11}$

Postoperative radiation therapy for clinical NO neck dissection or negative SLNB is disputed in the literature, especially for MCC localized in the head and neck. ${ }^{11,18}$ In the most recently published NCCN Guidelines for $\mathrm{MCC}^{9}$, PORT (50-56 $\mathrm{Gy}$ ) on the primary tumor side is recommended even with negative resection margins, but observation is considered an option when the primary tumor is small, widely excised, and without other risk factors, such as lymphovascular invasion or immune suppression. Postoperative radiation therapy on the draining nodal basin is not indicated after negative SLNBs, but is always indicated after neck dissection with multiple involved nodes. However, PORT seems to increase local control, but it has no significant impact on MCC-specific death and overall survival. ${ }^{11,19-21}$ Considering that the morbidity related to PORT treatment is generally worse for the head and neck region and the lack of benefits in terms of survival, we did not perform PORT even in some cases recommended by the last NCCN guidelines. Patient number 3, despite the multiple lymph nodes present at the parotid gland, after neck dissection, refused PORT. He had no other risk factors and at the last follow-up is free of disease 28 months after the neck dissection. Patient 4 did not receive 
PORT despite her immune disorders (RA, MGUS) because she was 88 years old and the MCC was near the eye. She had a local recurrence but is now free of disease ( 7 months after the second surgery and 26 months after the first surgery). Examining our patients and the literature we think that it is possible to avoid the morbidity of PORT in patients with small tumors radically resected with wide resection margins. If the follow-up is close, we think that in selected cases it is possible to avoid PORT even if they present some risk factors (to determine by means of further and bigger studies) without affecting the overall survival.

\section{Conclusion}

A strict follow-up is mandatory in patients with autoimmune disorders.

The parotid gland should be strictly controlled in patients with lower eyelid tumors and maybe we could consider superficial parotidectomy in these patients even if SLNs are located in a very peripheral part of the parotid gland.

In selected cases, it is possible to avoid the morbidity of PORT in patients with small size MCCs of the head and neck even if some risk factors are.

Conflict of Interests

The authors have no conflict of interests to declare.

\section{References}

1 Schadendorf D, Lebbé C, Zur Hausen A, et al. Merkel cell carcinoma: Epidemiology, prognosis, therapy and unmet medical needs. Eur J Cancer 2017;71:53-69

2 van der Zwan JM, Trama A, Otter R, et al; RARECARE WG. Rare neuroendocrine tumours: results of the surveillance of rare cancers in Europe project. Eur J Cancer 2013;49(11):2565-2578

3 Fitzgerald TL, Dennis S, Kachare SD, Vohra NA, Wong JH, Zervos EE. Dramatic increase in the incidence and mortality from Merkel cell carcinoma in the United States. Am Surg 2015;81(08):802-806

4 Youlden DR, Soyer HP, Youl PH, Fritschi L, Baade PD. Incidence and survival for Merkel cell carcinoma in Queensland, Australia, 19932010. JAMA Dermatol 2014;150(08):864-872

5 Becker JC, Stang A, DeCaprio JA, et al. Merkel cell carcinoma. Nat Rev Dis Primers 2017;3:17077

6 Becker JC. Merkel cell carcinoma. Ann Oncol 2010;21(Suppl 7): vii81-vii85
7 Lemos BD, Storer BE, Iyer JG, et al. Pathologic nodal evaluation improves prognostic accuracy in Merkel cell carcinoma: analysis of 5823 cases as the basis of the first consensus staging system. J Am Acad Dermatol 2010;63(05):751-761

8 Feng H, Shuda M, Chang Y, Moore PS. Clonal integration of a polyomavirus in human Merkel cell carcinoma. Science 2008;319 (5866):1096-1100

9 Rollison DE, Giuliano AR, Becker JC. New virus associated with merkel cell carcinoma development. J Natl Compr Canc Netw 2010;8(08):874-880

10 NCCN Guidelines Version 1.2018 Merkel Cell Carcinoma

11 Müller-Richter UDA, Gesierich A, Kübler AC, Hartmann S, Brands RC. Merkel Cell Carcinoma of the Head and Neck: Recommendations for Diagnostics and Treatment. Ann Surg Oncol 2017;24 (11):3430-3437

12 Filitis DC, Paragh G, Samie FH, Zeitouni NC. Sentinel lymph node biopsy in periocular merkel cell carcinoma: a case report. BMC Res Notes 2017;10(01):490

13 Smith FO, Yue B, Marzban SS, et al. Both tumor depth and diameter are predictive of sentinel lymph node status and survival in Merkel cell carcinoma. Cancer 2015;121(18):3252-3260

14 Kivelä T, Tarkkanen A. The Merkel cell and associated neoplasms in the eyelids and periocular region. Surv Ophthalmol 1990;35 (03):171-187

15 Iyer JG, Storer BE, Paulson KG, et al. Relationships among primary tumor size, number of involved nodes, and survival for 8044 cases of Merkel cell carcinoma. J Am Acad Dermatol 2014;70(04): 637-643

16 Schwartz JL, Griffith KA, Lowe L, Wong SL, McLean SA, Fullen DR, et al. Features predicting sentinel lymph node positivity in Merkel cell carcinoma. J Clin Oncol 2011;29(08):1036-1041

17 Fields RC, Busam KJ, Chou JF, et al. Recurrence and survival in patients undergoing sentinel lymph node biopsy for merkel cell carcinoma: analysis of 153 patients from a single institution. Ann Surg Oncol 2011;18(09):2529-2537

18 Deganello A, De Bree R, Dolivet G, Leemans CR. Infrahyoid myocutaneous flap reconstruction after wide local excision of a Merkel cell carcinoma. Acta Otorhinolaryngol Ital 2005;25(01): 50-53, discussion 53-54

19 Mojica P, Smith D, Ellenhorn JD. Adjuvant radiation therapy is associated with improved survival in Merkel cell carcinoma of the skin. J Clin Oncol 2007;25(09):1043-1047

20 Veness M, Howle J. Radiotherapy alone in patients with Merkel cell carcinoma: the Westmead Hospital experience of 41 patients. Australas J Dermatol 2015;56(01):19-24

21 Takagishi SR, Marx TE, Lewis C, et al. Postoperative radiation therapy is associated with a reduced risk of local recurrence among low risk Merkel cell carcinomas of the head and neck. Adv Radiat Oncol 2016;1(04):244-251 